\title{
THE EFFECT OF A CENTRAL MASSIVE BLACK HOLE ON THE GAS FUELING
}

\author{
H. FUKUDA AND A. HABE \\ Division of Physics, Hokkaido University \\ Japan \\ AND \\ K. WADA \\ National Astronomical Observatory \\ Japan
}

\section{Introduction}

Nuclear activities in galaxies, such as nuclear starbursts or AGNs, are supposed to be induced by gas fueling into nuclear regions of galaxies. Nonaxisymmetric gravitational potential caused by a stellar bar is a convincing mechanism for triggering gas fueling (Phinney 1994). However, numerical simulations have shown that the bar can not force the gas to accrete toward the galactic center beyond the inner Lindblad resonance (ILR). As a mechanism to overcome the ILR barrier, the double barred structure (Friedli \& Martinet 1993), or the self-gravity of gas (Wada \& Habe 1992, 1995; Elmegreen 1994) are proposed.

For an alternative mechanism to fuel gas to a galactic center, we investigate the effect of a central massive black hole $(\mathrm{CMBH})$. If the $\mathrm{CMBH}$ exists, an additional ILR (hereafter a nuclear Lindblad resonance (NLR)) appears inside of the usual ILRs. Therefore, the stellar and gas dynamics in the resonant region are affected by the NLR, and the gas fueling into the inside of the usual ILRs caused by the NLR is expected. Pfenniger \& Norman (1990) showed that the dissipation is enhanced in the resonance region and that the gas inflow is boosted inside the ILRs (including the NLR). However, since they used weakly dissipative single-particle to follow motion of idealised gas clouds, the effects of the NLR on the dynamics of the actual interstellar matter is not still clear. 


\section{Numerical simulations}

We investigate the dynamics of a gas disk near the NLR region in a weakly barred galaxy with the CMBH by using the smoothed particle hydrodynamics (SPH) method. Our gas disk model and code are based on the that of Wada \& Habe (1992). We assume that the gas is isothermal $\left(C_{\mathbf{s}} \sim 10 \mathrm{~km}\right.$ $\mathrm{s}^{-1}$ ) and non-self-gravitating, and that the mass of the $\mathrm{CMBH}$ is about 1 per cent of the mass of a galaxy. Initial gas disk is distributed within twice the radius of the NLR. The time evolution of the gas disk is as follows. At about one rotation period of gas disk, two trailing spiral shocks occur and extend to the edge of the gas disk. Then, the gas loses angular momentum substantially at the shocks and flows drastically into the center to make a gas ring with a radius of about one-third of that of the NLR. After about three rotation period of gas disk, almost all the gas has fallen into the gas ring. In the no-NLR case, such a rapid fueling does not occur.

\section{Discussion}

We have made numerical simulation of the gas disks around a CMBH in a weakly barred potential and have shown the effects of the NLR caused by the CMBH. We have shown that the gas is highly disturbed by the NLR and trailing spiral shocks are formed and the gas is finally accumulated into the ring around the CMBH whose radius is about one-third of that of the NLR.

In our simulations, the nuclear gas ring and the spiral shocks associated with the ring are formed. Similar gas distribution is observed in nuclear region of many active galaxies and starburst galaxies (Kenny 1993 for a review). In the gas accumulation phase in to the gas ring, violent gas motion is excited and strong shocks occur in the nuclear region. Since massive gas concentrates in these region, strong star formation is expected during this phase. Our numerical results can explain star formation activity in these galaxies, and we suggest that our gas fueling mechanism can be related to the fueling mechanism for AGNs.

\section{References}

Elmegreen B. G., 1994, ApJ, 425, L73

Friedli D., Martinet L., 1993, A\&A, 277, 27

Kenny J. D. P., 1993, Mass Transfer Induced Activity in Galaxies, ed, I. Shlosman, Cambridge Univ. Press, Cambridge, p.78-89

Pfenniger D., Norman C. A., 1990, ApJ, 363, 391

Phinney E. S., 1994, Mass Transfer Induced Activity in Galaxies, ed, I. Shlosman, Cambridge Univ. Press, Cambridge, p.1-22

Wada K., Habe A., 1992, MNRAS, 258, 82

Wada K., Habe A., 1995, MNRAS, 277, 433 